\title{
Bio-inspired topological skeleton for the analysis of quadruped kinematic gait
}

\author{
Benjamin BOUDON ${ }^{1}$, Jean-Marc LINARES ${ }^{1}$, Anick ABOURACHID ${ }^{2}$, Arthur VAUQUELIN ${ }^{1}$, Emmanuel \\ MERMOZ ${ }^{1,3}$ \\ 1. Aix Marseille Univ, CNRS, ISM, Marseille, France \\ 2. Muséum National d'Histoire Naturelle, Pavillon d'Anatomie Comparée, 57 rue Cuvier, F-75231 Paris Cedex 05, France \\ 3. Airbus Helicopters, Aéroport de Marseille Provence, 13700 Marignane, France
}

\begin{abstract}
In bio-inspired design activities, Nature is a basis of knowledge. Over the last twenty years, many solutions to measure and analyze human or animal gaits have been developed (VICON system, X-ray radiography...). Although, these methods are becoming more and more accurate, they are quite expensive, long to set up and not easily portable. In this paper, a method called the bio-inspired topological skeleton is suggested in order to complement the classic videography process and to enable animal gait analysis. A new predictive kinematic model with closed-loops of an unguligrade quadruped will be suggested. This kinematic model includes three segments per leg and takes into account the scapula movements. The proposed method allows us to improve the accuracy of the kinematic input data measured from a single video including an additional artefact. To show the benefits of this method, joint parameters that are difficult to measure are derived symbolically from a kinematic model and compared with experimental data.
\end{abstract}

Keywords: Quadruped locomotion, Motion analysis, Topological Skeleton, Closed-form solutions, Multibody systems (MBS)

\section{Introduction}

The gait analysis is a scientific activity which is quite old and which started in the 17th century with the visual analysis of human locomotion conducted by Borelli ${ }^{[1]}$. Later, the first device, the famous photographic gun used to study the gait kinematic, was created by Marey ${ }^{[2,3]}$ in Paris. The idea of his method, known as chronophotography, was to analyse the motion from a series of still-pictures. Muybridge ${ }^{[4]}$ used this technique in 1902 to analyse the gait of horses running at normal speed. Later, the $2 \mathrm{D}$ video system has also continued to improve thanks to the use of the motion picture camera ${ }^{[5]}$ and high-speed motion picture camera ${ }^{[6]}$. At the end of the twentieth century, the motion analysis system became automated with the development of videos and computers. 3D video systems such as VICON ${ }^{[7]}$ or CODA ${ }^{[8]}$ have allowed the use of passive markers and the motion could also be easily recorded on videos. Finally, some rare studies used the $\mathrm{x}$-ray cinema to analyze the skeleton during the activity of animals ${ }^{[9-13]}$ and to get the accurate bone positions during the locomotion. Other techniques for locomotion analysis exist, such as accelerometry ${ }^{[14]}$, electromyography (EMG) ${ }^{[15]}$ and goniometry ${ }^{[16]}$. However, in this paper, only techniques purely based on kinematic principles have been considered in our scope. The « high-precision » techniques mentioned previously (3D motion analysis or x-ray cinema) can have some drawbacks in terms of cost and full portability or accessibility. First, these methods always need a preliminary preparation of the experiment. A direct contact with the subject is needed and the passive markers should be initially fixed to the studied subject. A calibration phase is also a mandatory phase except for the latest 3D motion systems such as CODA ${ }^{[17]}$. Secondly, these modern devices are very expensive, and the cost of the experimentation phases continue to grow when many different species are studied. As far as the « standard $2 \mathrm{D}$ video system is concerned, it always needs a preliminary preparation step for positioning pre-markers on the studied subject. Consequently, the direct use of gait videos which haven't been preliminarily "marked", or where the video quality has become degraded, or where some parts of the still-picture present a loss of resolution due to a quick movement, is almost impossible to conduct. In the frame of bio-inspiration research from living beings ${ }^{[18]}$, the observation of animals in any natural environment is essential but access to them is often very limited. As mentioned in [19], video analysis present an attractive measurement technique which requires "little to no manipulation or instrumentation of the animal to be measured". In this paper, a method using a topological skeleton is suggested to help the experimenter obtain better measurements from a standard video process of animal gait. An additional artefact is included as a geometric standard to calibrate the global video process and improve the method's accuracy. The benefit of this method is to provide a portable and low-cost process that measures the animal's gaits. Moreover, this method 
doesn't require any markers or sensors and is easy to conduct. The evolution of devices and technology from everyday life such as smart phones and motion picture cameras allow us to create films easily, making this aspect easier and convenient. This method has become possible thanks to the evolution of Computer Aided Design (CAD) software ${ }^{[20]}$. The idea of this method is to give the experimenter more physical information linked to the geometry and the structure of the studied subject thanks to a bio-inspired model that is called "bio-inspired topological skeleton" (BITS) in this paper. The presented topological skeleton built with a CAD sketch and used as pattern positioned on still-pictures will help the experimenter to measure positions and angles by taking into account geometric and topological constraints deduced from the morphologic analysis of an animal. In the state of the art, the consideration of scapula movement is not included in current research gait studies. In this paper, a new predictive kinematic model with closed-loops of an unguligrade quadruped will be proposed. This kinematic model includes three segments per leg and mostly, takes into account the scapulae movements. For example, even in these recent quadruped robots ${ }^{[21-23]}$, no scapula model and its associated linkage are used. The positional parameters which define the model are partitioned into inputs (independent joint parameters) and outputs (dependent joint parameters). Once the inputs are determined with the skeleton method, joint parameters that are difficult to measure will be estimated from a kinematic model and compared with experimental data to show the benefits of the method. The resolution of the kinematics has been conducted by solving symbolically the constraints equations. As pointed in ${ }^{[24]}$, this method needs numerous symbolic manipulation. However, this work made on the geometry facilitates further developments such as the mechanical dynamics behavior of bio-inspired mechanisms or robots ${ }^{[25]}$. The gait analysis method suggested in this paper is summed up in Fig 1 . The kinematic input data denoted $\mathbf{p}_{\mathbf{i}}$ and the measured outputs $\mathbf{p}_{\mathbf{o}}$ with the BITS on still-pictures are first collected. Then, the kinematic model allows the calculation of an estimation of the kinematic outputs $\hat{\mathbf{p}}_{\mathbf{0}}$. The calculated kinematic outputs $\hat{\mathbf{p}}_{\mathbf{0}}$ will then be compared with the measured output $\mathbf{p}_{\mathbf{0}}$. The notation used will be detailed in Section 4 .

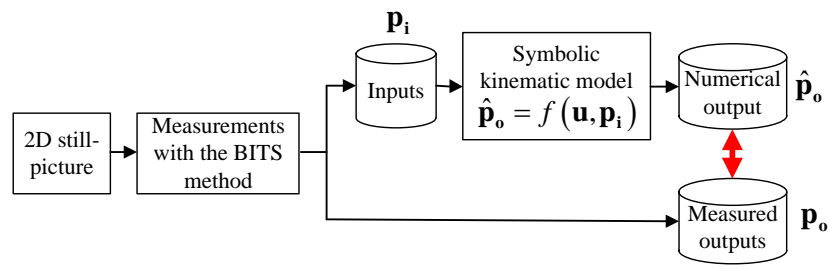

Fig 1 Gait analysis method

The paper is organized as follows. In section 2, the BITS and its scientific foundations are presented. Then, a BITS for an unguligrade quadruped is determined based on a proposition of a kinematic model. The mechanical assumptions which lead to this model have been chosen from the morphology analysis of the unguligrade quadruped. Section 3 details the calibration process and the use of the BITS on a video of quadruped. Section 4 presents a symbolic resolution of kinematic problems. Simulation results and a validation based on experimental data which consist in a video of sheep will be presented in Section 5.

\section{Bio-inspired topological skeleton}

\subsection{Unguligrade quadruped kinematic model}

Following the morphological description detailed in ${ }^{[26]}$, the unguligrade skeleton has been decomposed into 3 groups: the axial skeleton, the appendicular skeleton and the appendages. The different parts of the skeleton will be illustrated on the sheep skeleton in Fig 2. The axial skeleton is composed of the head and the vertebral column which is made up of vertebrae. In quadrupeds, the vertebral column is differentiated into the neck, the dorsal region, the sacral region and the tail region. The appendicular skeleton is composed of the pectoral and pelvic girdles on which the limbs are articulated. For ungulates, the pectoral girdle consists of a pair of scapulae, with a muscular link with the axial skeleton. The scapula movement during locomotion moves the shoulder joint forward and backward on which the forelimb is articulated. The pelvis is strongly fixed on the axis via the sacrum and is not mobile with regard to the axial 
skeleton. Consequently, the pelvic girdle will be considered as a part of the axial skeleton. The tetrapod limb consists of three parts: the stylopod with, for the fore and hind limbs respectively, the humerus and the femur bones; the zeugopod, with the radius cubitus and tibia-fibula bones and the autopod with the carpus, metacarpus and phalanges for the hand, and the tarsus, metatarsus and phalanges for the foot. The bones of the autopod may be more or less fused depending on the species, the higher degree of fusion between the bones are found in the unguligrades like the sheep. For that reason, the autopod will be considered as one segment in this study.

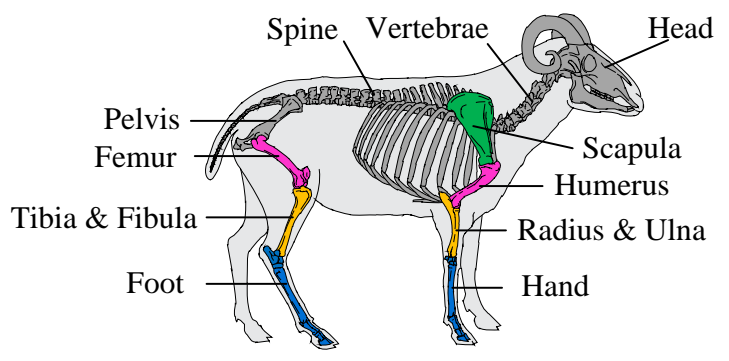

Fig 2 Sheep skeleton structure

From the previous morphologic analysis and the 2D video experimental setup which has been used and which will be presented in Section 3, different assumptions on bodies, joints and kinematics are done. In this study, only rigid bodies are taken into consideration. The head and the axis plus pelvis bones, are considered as one rigid body which is called the rigid axial skeleton in the rest of the paper. The scapulae are considered to be two rigid bodies. The appendages are composed of two hind limbs and two forelimbs. For the hind limbs, every limb is decomposed as three rigid bodies: the femur, the tibia-fibula and the foot considered to be the merger of the autopod. For the fore limbs, every limb is divided into three rigid bodies also: the humerus, the radius-ulna and the hand considered to be the merger of the autopod. The terminology of the rigid bodies considered are given in Figure 3. Classically, the definition of the kinematic joints for the hind limbs and forelimbs as reviewed in ${ }^{[27]}$ are the follows: shoulder and hip joints can be considered as spherical joints, elbow and knee joints can be considered as specific joints (revolution + gliding), wrist and ankle joints can be considered as revolute joints. During locomotion in a straight trajectory, the joints' amplitudes of movement in the frontal and in the transverse planes are insignificant compared to the joint's amplitudes of movement in the sagittal plane. As an example, in the knee, flexion/extension amplitude, considered as a primary motion, is higher than abduction/adduction or internal/external amplitude which are considered as secondary motions like anterior/posterior translation. Due to the amplitude of joints in the frontal and in the transverse planes and due the process of measurement, all previous joints are considered as revolute joints except the mechanical joint between the trunk and the scapula, which has been modelled with a revolute joint associated with a prismatic joint. The assumption made about the joints are illustrated in Fig 3. The movements of the four legs remain parallel to the sagittal plane or plane of symmetry of the unguligrade. Consequently, the global movement could be described by a 2D kinematic model instead of a 3D kinematic model (Fig 3).

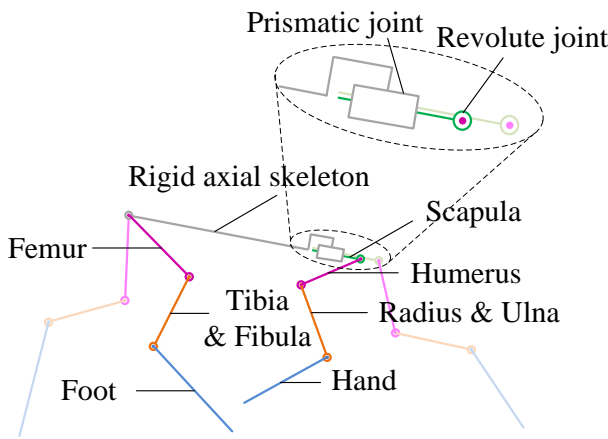

Fig 3 Assumptions leading to the 2D kinematic model 
Finally, these assumptions lead to a well-defined framework in which the method presented in this paper can give accurate results. of bionic engineering journal of bionic engineering.

\subsection{CAD implementation of a bio-inspired skeleton}

For the CAD design, the BITS concept has been created in order to localize a set of mechanism points in space ${ }^{[20,}$ ${ }^{28,29]}$. Using a BITS for gait analysis provides information on geometric constraints and topological constraints during the gait measurement phase. In 2D, the geometric constraints consist in the three following, defined from two elementary objects (vectors and oriented angles).

- Distance between two points on the same segment is expressed by:

$$
\operatorname{dist}\left(M_{1}, M_{2}\right)=\left\|\mathbf{M}_{1} \mathbf{M}_{2}\right\|=\text { Cte }
$$

- Points coincidence: two points $\mathrm{M}_{1}$ and $\mathrm{M}_{2}$ which have the same spatial position are defined as coincident.

$$
M_{1}=M_{2}
$$

- Lines coincidence: two lines $\mathrm{D}_{1}$ and $\mathrm{D}_{2}$ have the same direction unit vector and the distance $\mathrm{d}$ between them is null. These two constraints are expressed by equation (3) and equation (4).

$$
\mathbf{t}_{\mathbf{1}}=\mathbf{t}_{\mathbf{2}}
$$

and

$$
d=\left\|\mathbf{M}_{1} \mathbf{M}_{2} \times \mathbf{t}_{1}\right\|=0
$$

with the direction unit vector of the line $\left(D_{i}\right)$ and $M_{i}$ a point of the line $\left(D_{i}\right)$.

Based on the quadruped kinematic model suggested in the previous section, a BITS skeleton is defined by 10 geometric constraints of two types: points coincidences and lines coincidences and 10 geometric parameters.

Table 1 Geometrical constraints

\begin{tabular}{ll}
\hline \multicolumn{1}{c}{ Constraints definition } & Constraints type \\
\hline $\mathrm{A}_{\mathrm{i}}-\mathrm{Fo}=\mathrm{A}_{\mathrm{i}}-\mathrm{TF}=\mathrm{A}_{\mathrm{i}}$ with $i=1 . .2$ & Points coincidence \\
$\mathrm{B}_{\mathrm{i}}-\mathrm{TF}=\mathrm{B}_{\mathrm{i}}-\mathrm{Fe}=\mathrm{B}_{\mathrm{i}}$ with $i=1 . .2$ & Points coincidence \\
$\mathrm{C}_{\mathrm{i}}-\mathrm{Fe}=\mathrm{C}_{\mathrm{i}}-\mathrm{RAS}$ with $i=1 . .2$ & Points coincidence \\
$\mathrm{C}_{4} \mathrm{C}_{2} / / \mathrm{C}_{4} \mathrm{D}_{1}$ & Lines coincidence \\
$\mathrm{C}_{4} \mathrm{C}_{2} / / \mathrm{C}_{4} \mathrm{D}_{2}$ & Lines coincidence \\
$\mathrm{D}_{\mathrm{i}}-\mathrm{Sca}=\mathrm{D}_{\mathrm{i}}-\mathrm{Hu}$ & Points coincidence \\
$\mathrm{B}_{\mathrm{i}}-\mathrm{Hu}=\mathrm{B}_{\mathrm{i}}-\mathrm{RU}$ & Points coincidence \\
$\mathrm{A}_{\mathrm{i}}-\mathrm{RU}-\mathrm{A}_{\mathrm{i}}-\mathrm{H}$ & Points coincidence \\
\hline
\end{tabular}

In the standard process of the video analysis, the identification of the markers is made either by a human operator or by an automatic process in a software based on a two-dimension cross-correlation of a set of pixels ${ }^{[19]}$. However, whatever the selected method for the point's identification, all the point measurements are positioned independently. Consequently, an error in a point's measurement can lead to a segment being very distant from the rigid axial body. With the BITS, the geometrical constraints guarantee the fact that the points should be consistent with constraints imposed by the kinematic joint's mechanism as illustrated in Fig 4. The geometric constraints are defined in Fig. 1.

With a standard process, the length of each segment can vary depending on the different configurations which are considered. The geometric parameters illustrated in Figure 5 guarantee that each segment stays rigid and thus preserves a constant length. The geometric parameters are defined respectively in Table 2. 


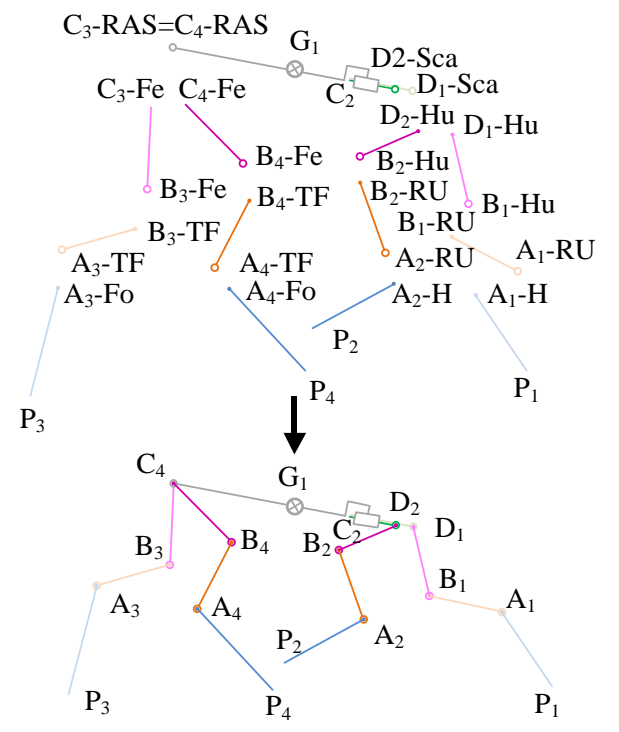

Fig 4 Geometrical constraints

Table 2 Geometric parameters

\begin{tabular}{lcc}
\hline \multicolumn{1}{c}{ Bodies } & Definition & $\begin{array}{c}\text { Geometric } \\
\text { parameters }\end{array}$ \\
\hline Rigid axial skeleton & $\mathrm{C}_{4} \mathrm{G}_{1}$ & $\mathrm{l}_{\mathrm{r}}$ \\
& $\mathrm{C}_{2} \mathrm{G}_{1}$ & $\mathrm{l}_{\mathrm{f}}$ \\
Humerus (or front stylopod) & $\mathrm{D}_{1} \mathrm{~B}_{1}=\mathrm{D}_{2} \mathrm{~B}_{2}$ & $\mathrm{~s}_{\mathrm{f}}$ \\
Radius-Ulna (or front zeugopod) & $\mathrm{B}_{1} \mathrm{~A}_{1}=\mathrm{B}_{2} \mathrm{~A}_{2}$ & $\mathrm{Z}_{\mathrm{f}}$ \\
Hand (or front autopod) & $\mathrm{A}_{1} \mathrm{P}_{1}=\mathrm{A}_{2} \mathrm{P}_{2}$ & $\mathrm{a}_{\mathrm{f}}$ \\
Femur (or rear stylopod) & $\mathrm{C}_{3} \mathrm{~B}_{3}=\mathrm{C}_{4} \mathrm{~B}_{4}$ & $\mathrm{~S}_{\mathrm{r}}$ \\
Tibia-Fibula (or rear zeugopod) & $\mathrm{B}_{3} \mathrm{~A}_{3}=\mathrm{B}_{4} \mathrm{~A}_{4}$ & $\mathrm{Z}_{\mathrm{r}}$ \\
Foot (or rear autopod) & $\mathrm{A}_{3} \mathrm{P}_{3}=\mathrm{A}_{4} \mathrm{P}_{4}$ & $\mathrm{a}_{\mathrm{r}}$ \\
\hline
\end{tabular}

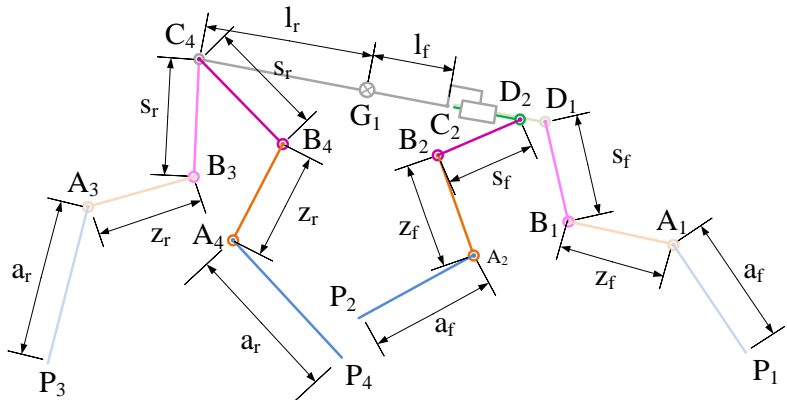

Fig 5 Geometric parameters

\section{Kinematic data measurements using a BITS}

\subsection{Experimental setup and calibration}

Kinematic data come from a study on the sheep locomotion ${ }^{[30]}$. The sheep moved in a 3-meter-wide and 15 meter long passage. The motion picture camera was placed on a tripod placed half-way down the passage 3 meters from the center thus ensuring a 3-meter stretch of passage could be filmed. The experimental setup includes an optical artefact composed with markings on the ground (interdistance W and depth D) and calibrated lengths on a barrier (interdistance $\mathrm{W}$ and height $\mathrm{H}$ ) in Fig 6. One clinically healthy intact sheep was used for this kinematic study. In this process, a vision calibration model has been used so as to determine the link between the coordinates $[u, v]$ of a point on the still picture and the coordinates $[x, y, z]$ of a point $\mathrm{M}$ of the real object. This model takes into account the internal geometric and optical camera characteristics classically described with the pinhole model (intrinsic parameters) and the positioning of 
the camera frame $\mathrm{R}_{\mathrm{c}}$ relative to a certain world coordinates system $\mathrm{R}_{0}$ (extrinsic parameters). This calibration process similar to the one used in ${ }^{[31]}$ has been conducted so as to determine the parameters associated to the transformation between the coordinates $[u, v]$ of the still-picture and the coordinates of the real object $[x, y, z]$. The vision calibration model (including distorsion as described in ${ }^{[32]}$ ) consisted in a calibration matrix with 10 parameters. Their identification were derived by a set of $\mathrm{M}_{\mathrm{k}}\left(x_{k}, y_{k}, z_{k}\right)$ points with $k=1 . .18$. These points are positioned on an 3D artefact (18 points on a barrier and markings on the ground as it is shown on the Fig 6). The parameters were derived using the least-squares method.

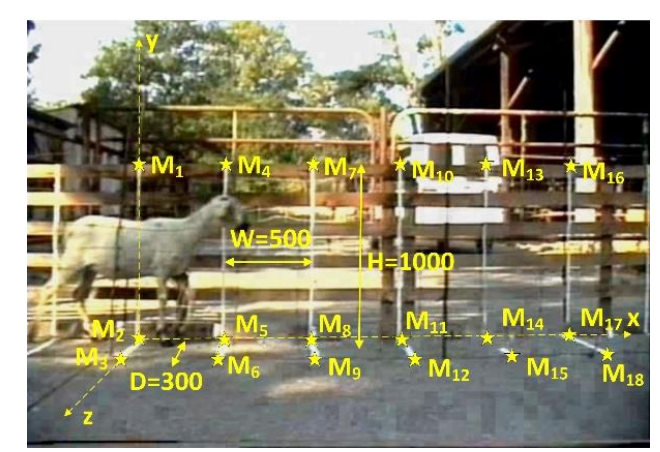

Fig 6 Calibration process

In this method, the motion picture camera is expected to stay parallel to the sagittal plane of the moving sheep. This assumption has enabled to deduce the depth dimension. It can be noticed that an acquisition process with two motion picture cameras could enable to circumvent the lack of information of the depth dimension.

\subsection{Identification of the geometric sheep dimensions}

The video is divided into 24 separate still-pictures. The method for identifying the geometric sheep dimensions is based on two steps. The first step is the calculation of the lengths of limb segments for one picture. For each picture, the $3 \mathrm{D}$ estimated coordinates $[x, y, z]$ of a point in the world coordinates system $\mathrm{R}_{0}$ have been derived from the $2 \mathrm{D}$ still-picture coordinates $[u, v]$ using a compensation process. This process used the inverse of the previous calibration matrix. The lengths of limb segments have been calculated from the norm of two end points of the physical segments. The Guide Uncertainties Measurements (GUM) ${ }^{[33]}$ is used to determine the uncertainties associated to the measurement process through the use of covariance matrices and jacobian matrices. In the second step, the lengths are then being averaged for all the pictures used. The mean value and measurement relative uncertainty are given in Table 3.

Table 3 Geometric sheep data

\begin{tabular}{lccc}
\hline \multicolumn{1}{c}{ Body } & $\begin{array}{c}\text { Geometric } \\
\text { parameter }\end{array}$ & $\begin{array}{c}\text { Mean } \\
\text { value } \\
(\mathrm{mm})\end{array}$ & $\begin{array}{c}\text { Measurement } \\
\text { relative } \\
\text { uncertainty }(\%)\end{array}$ \\
\hline Rigid axial skeleton & $\mathrm{l}_{\mathrm{r}}$ & 335,2 & 5.64 \\
Humerus & $\mathrm{l}_{\mathrm{f}}$ & 295,5 & 5.60 \\
RadiusUlna & $\mathrm{s}_{\mathrm{f}}$ & 155,5 & 4.44 \\
Hand & $\mathrm{z}_{\mathrm{f}}$ & 210,3 & 6.27 \\
Femur & $\mathrm{a}_{\mathrm{f}}$ & 248,4 & 6.20 \\
TibiaFibula & $\mathrm{s}_{\mathrm{r}}$ & 197,5 & 6.19 \\
Foot & $\mathrm{z}_{\mathrm{r}}$ & 213,4 & 3.28 \\
\hline & $\mathrm{a}_{\mathrm{r}}$ & 274,6 & 5.35 \\
\hline
\end{tabular}




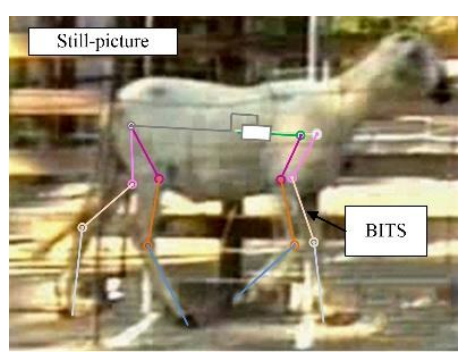

Fig 7 Use of the CAD BITS on a still-picture

\subsection{Practical process for the data measurements with a BITS}

These geometric sheep data (Table 3) have been used to set the values of the geometric parameters of the BITS (Table 2). By using the compensation process, the pictures are also translated in the 3D space in the sketcher of a CAD software (Fig 7). The BITS is used with the following method. First, drag the extremities of the BITS to the hooves of the studied subject in the still-picture. Second, align the autopods' rigid bodies (in blue in Fig 7) to the autopods in the still-picture, third, once the position and orientation of these first bodies are appropriate, lock them in place. Next, proceed similarly for the zeugopods. These bodies have a rotational movement since the bottom extremities of these bodies are constrained by the points coincidence constraints of the BITS. Finally, apply the same procedure for the remaining rigid bodies. The leg crosses are managed by interpolation with the previous and following configurations.

\subsection{Comparison between data obtained by the BITS and standard method}

The BITS method is compared to the standard method to acquire data from a video. The standard method is based on the acquisition of points and angles from a still-picture. In other words, with standard video analysis, the post-markers are positioned independently of the geometry and topology of the studied subject. The variation of the length segment which can occur with the standard method is shown in Fig 8 on the example of the femur. A maximum deviation of $37 \mathrm{~mm}$ can be found between the standard method and the BITS method.

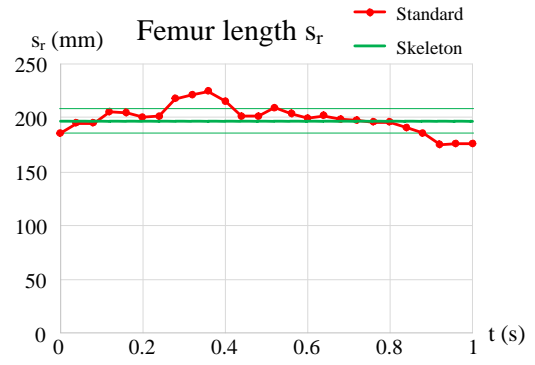

Fig 8 Evolution of the measurements of the femur length

A comparison is now presented on the positional parameters. The kinematics' angle inputs used in the kinematic models will be the angle between the first segment with the floor $\left(\gamma_{i}(t)\right.$ with $\left.i=1 \ldots 4\right)$ and the ankle/ wrist angle $\left(\phi_{i}(t)\right.$ with $i=1 . .4$ ). The definition of theses parameters will be explained in Section 0 . These positional parameters are located at the bottom of the limb and have been chosen as input data because they are the easiest to measure. These angles have been measured both with the standard method and the BITS method. The results are shown only for the forelimb for sake of concision. It is observable that the green curve obtained with the BITS method is smoother and consequently with less discontinuities than the red curve obtained with the standard method. 


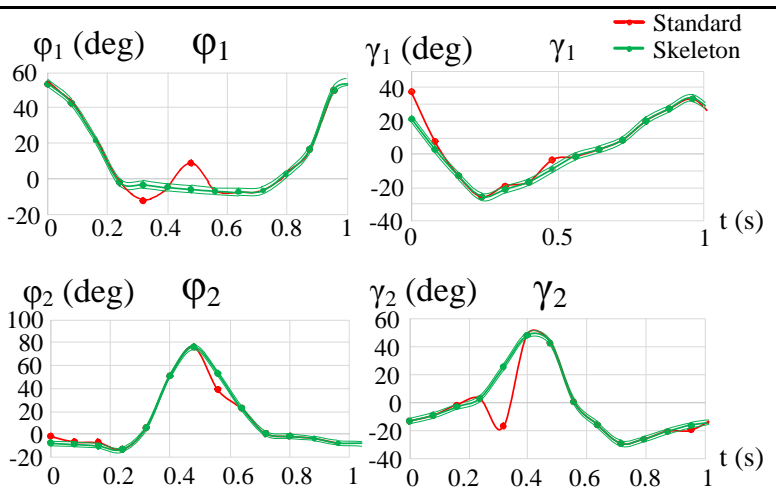

Fig 9 Inputs with standard and BITS methods (forelimbs)

\subsection{Discussion}

In some cases, too many errors on one measurement could occur because of the degraded quality of the still-picture, for example, with a quick movement of the subject. In the standard method, an inconsistent measurement with regard to the mechanism considered can lead to geometrical discontinuities. The issue comes from the fact that the measurement's points are conducted independently of the real geometry and real topology of the studied subject. For example, a constant length for each bone segment is not guaranteed. The BITS method reduces these errors. Indeed, the BITS method enables us to reduce the margin of error for those uncertain measurements due to the fact that the BITS allows the user to be guided to the biological solutions permitted by the mechanism.

\section{Kinematic gait estimation}

The previous method has allowed for more efficient measurements of some kinematic data. However, not all the kinematic data is easily measurable. For example, the kinematic parameters associated with the top of the quadruped's limbs are hidden by larger muscles. In this section, a kinematic model with closed kinematic loops is defined. The resolution of the topological constraints traditionally called the positional constraints equation in the mechanisms theory allowed us to estimate those parameters. The kinematic solution is obtained symbolically, and closed form solutions are derived. This will facilitate the study of the gait dynamics.

\subsection{Parametric kinematic scheme}

The studied unguligrade animal was modelled by a multibody system of 15 bodies which are assumed to be rigid. The system consists of a rigid axial skeleton, two forelegs which are each composed of 4 bodies: a scapula, a humerus, a radius, a hand and two hind legs which are each composed of 3 bodies: a femur, a tibia, a foot. $R_{0}=\left(O, \mathbf{x}_{0}, \mathbf{y}_{0}, \mathbf{z}_{\mathbf{0}}\right)$ is the global inertial reference frame to which position and orientation of all the bodies are referred. Some local moving reference frames are attached to these bodies. $R_{1}=\left(G, \mathbf{x}_{1}, \mathbf{y}_{1}, \mathbf{z}_{1}\right)$ is attached to the rigid axial skeleton. $R_{2}{ }^{i}=\left(D_{i}, \mathbf{x}_{2}{ }^{\mathrm{i}}, \mathbf{y}_{2}{ }^{\mathrm{i}}, \mathbf{z}_{2}{ }^{\mathrm{i}}\right)$ with $\mathrm{i}=1 . .2$ and $R_{2}{ }^{i}=\left(C_{i}, \mathbf{x}_{2}{ }^{\mathrm{i}}, \mathbf{y}_{2}{ }^{\mathrm{i}}, \mathbf{z}_{2}{ }^{\mathrm{i}}\right)$ with $\mathrm{i}=3 . .4$ are attached to the stylopods (respectively to humerus and femurs), $R_{3}{ }^{i}=\left(B_{i}, \mathbf{x}_{\mathbf{3}}{ }^{\mathbf{i}}, \mathbf{y}_{\mathbf{3}}{ }^{\mathbf{i}}, \mathbf{z}_{\mathbf{3}}{ }^{\mathrm{i}}\right)$ with $\mathrm{i}=1 . .4$ are attached to zeugopods (radius or tibia), $R_{4}{ }^{i}=\left(A_{i}, \mathbf{x}_{\mathbf{4}}{ }^{\mathrm{i}}, \mathbf{y}_{\mathbf{4}}{ }^{\mathrm{i}}, \mathbf{z}_{\mathbf{4}}{ }^{\mathrm{i}}\right)$ with $\mathrm{i}=1 . .4$ are attached to the autopods (hand or foot). The kinematic scheme is shown in Fig 10. 


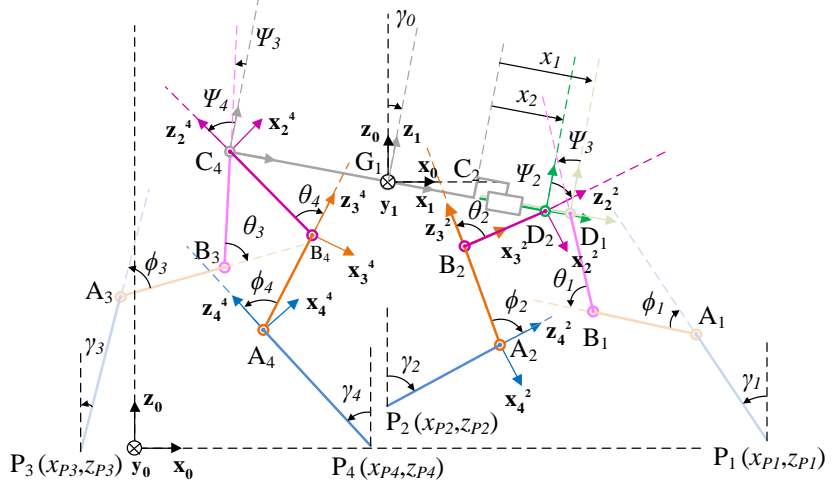

Fig 10 Kinematic scheme

All the positional parameters of each kinematic joint are defined in Table 4. Its are labelled: $\mathrm{F}$ for free joint, $\mathrm{P}$ for prismatic joint and $\mathrm{R}$ for revolute joint. All the positional parameters will be grouped in vector $\mathrm{p}$.

Table 4 Positional parameters

\begin{tabular}{lccc}
\hline \multicolumn{1}{c}{ Joint } & Type & \multicolumn{1}{c}{$\begin{array}{c}\text { Positional } \\
\text { parameters }\end{array}$} & $\begin{array}{c}\text { Total of } \\
\text { parameters }\end{array}$ \\
\hline $\begin{array}{l}\text { Rigid axial skeleton/ Ground } \\
\text { Scapula/ Rigid axial skeleton }\end{array}$ & $\mathrm{F}$ & $\mathrm{x}_{0}(\mathrm{t}), \mathrm{z}_{0}(\mathrm{t}), \gamma_{0}(\mathrm{t})$ & 3 \\
$\begin{array}{l}\text { Shoulder joint } \\
\text { (Humerus/ Scapula) }\end{array}$ & $\mathrm{P}$ & $\mathrm{x}_{\mathrm{i}}(\mathrm{t})$ with $\mathrm{i}=1 \ldots 2$ & 2 \\
$\begin{array}{l}\text { Elbow joint } \\
\text { (Cubitus-Radius/ Humerus) }\end{array}$ & $\mathrm{R}$ & $\theta_{\mathrm{i}}(\mathrm{t})$ with $\mathrm{i}=1 \ldots 2$ & 2 \\
$\begin{array}{l}\text { Wrist joint } \\
\begin{array}{l}\text { (Hand/ Cubitus-Radius) } \\
\text { Hand/ Ground }\end{array}\end{array}$ & $\mathrm{R}$ & $\phi_{\mathrm{i}}(\mathrm{t})$ with $\mathrm{i}=1 \ldots 2$ & 2 \\
\hline $\begin{array}{l}\text { Hip joint } \\
\text { (Femur/ Rigid axial skeleton) }\end{array}$ & $\mathrm{F}$ & $\mathrm{x}_{\mathrm{Pi}}(\mathrm{t}), \mathrm{z}_{\mathrm{Pi}}(\mathrm{t}), \gamma_{\mathrm{i}}(\mathrm{t})$ with $\mathrm{i}=1 . .2$ & 6 \\
$\begin{array}{l}\text { Knee joint } \\
\text { (Tibia-Perone/ Femur) }\end{array}$ & $\mathrm{R}$ & $\psi_{\mathrm{i}}(\mathrm{t})$ with $\mathrm{i}=3 \ldots 4$ & 2 \\
$\begin{array}{l}\text { Ankle joint } \\
\text { (Foot/ Tibia-Perone) }\end{array}$ & $\mathrm{R}$ & $\phi_{\mathrm{i}}(\mathrm{t})$ with $\mathrm{i}=3 \ldots 4$ & 2 \\
\begin{tabular}{l} 
Foot/ Ground \\
\hline Total number of positional parameters $\mathrm{i}=3 \ldots 4$
\end{tabular} & 2 \\
\hline
\end{tabular}

\subsection{Kinematic constraint equations}

The topological constraints are obtained by writing the position vector's loop closure (called, in the rest of the paper: vectorial loop) and the angular loop of four loops of bodies. The four loops considered are associated with the four appendages and each loop is closed by moving through the free joint between the rigid axial skeleton and the floor. The closed vectorial loop is a sequence of bipoints equal to the null vector. The angular loop is a Chasles's relation between the angles in closed loop. For the forelimbs, the vectorial loop gives for $\mathrm{i}=1 \ldots 2$ :

$\mathbf{O G}_{1}+\mathbf{G}_{1} \mathbf{C}_{\mathbf{i}}+\mathbf{C}_{\mathbf{i}} \mathbf{D}_{\mathbf{i}}+\mathbf{D}_{\mathbf{i}} \mathbf{B}_{\mathbf{i}}+\mathbf{B}_{\mathbf{i}} \mathbf{A}_{\mathbf{i}}+\mathbf{A}_{\mathbf{i}} \mathbf{P}_{\mathbf{i}}+\mathbf{P}_{\mathbf{i}} \mathbf{O}=\mathbf{0}$

For the forelimbs, the angular loop gives for $\mathrm{i}=1 \ldots 2$ :

$$
\left(\mathbf{z}_{\mathbf{0}}, \mathbf{z}_{\mathbf{1}}\right)+\left(\mathbf{z}_{\mathbf{1}}, \mathbf{z}_{\mathbf{2}}{ }^{\mathbf{i}}\right)+\left(\mathbf{z}_{\mathbf{2}}, \mathbf{z}_{\mathbf{3}}{ }^{\mathrm{i}}\right)+\left(\mathbf{z}_{\mathbf{3}}{ }^{\mathrm{i}}, \mathbf{z}_{\mathbf{4}}{ }^{\mathbf{i}}\right)+\left(\mathbf{z}_{\mathbf{4}}{ }^{\mathrm{i}}, \mathbf{z}_{\mathbf{0}}\right)=\mathbf{0}
$$

For the hindlimbs, the vectorial loop gives for $\mathrm{i}=3 \ldots 4$ :

$$
\mathbf{O G}_{1}+\mathbf{G}_{1} \mathbf{C}_{\mathbf{i}}+\mathbf{C B}_{\mathbf{i}}+\mathbf{B}_{\mathbf{i}} \mathbf{A}_{\mathbf{i}}+\mathbf{A}_{\mathbf{i}} \mathbf{P}_{i}+\mathbf{P}_{\mathbf{i}} \mathbf{O}=\mathbf{0}
$$

For the hindlimbs, the angular loop gives for $\mathrm{i}=3 \ldots 4$ :

$$
\left(\mathbf{z}_{\mathbf{0}}, \mathbf{z}_{\mathbf{1}}\right)+\left(\mathbf{z}_{\mathbf{1}}, \mathbf{z}_{\mathbf{2}}{ }^{\mathbf{i}}\right)+\left(\mathbf{z}_{\mathbf{2}}{ }^{\mathrm{i}}, \mathbf{z}_{\mathbf{3}}{ }^{\mathrm{i}}\right)+\left(\mathbf{z}_{\mathbf{3}}{ }^{\mathrm{i}}, \mathbf{z}_{\mathbf{4}}{ }^{\mathbf{i}}\right)+\left(\mathbf{z}_{\mathbf{4}}{ }^{\mathbf{i}}, \mathbf{z}_{\mathbf{0}}\right)=\mathbf{0}
$$

In 2D, starting from a closed loop, for each loop, 3 equations can be written: two equations obtained by projecting the vectorial loop on two orthogonal vectors and the equation coming from the angular loop. To make the equation 
shorter, cos and sin are replaced by $\mathrm{C}$ and $\mathrm{S}$ and the time dependency is written by using bold characters. It leads to the following set of $2 \times 6$ equations:

For the forelegs $(\mathrm{i}=1 \ldots 2)$ :

$$
\begin{gathered}
\boldsymbol{x}_{\boldsymbol{\theta}}-\boldsymbol{x}_{\boldsymbol{P}_{i}}+\mathrm{C}\left(\gamma_{0}\right) l_{f}-S\left(\gamma_{0}+\boldsymbol{\psi}_{i}\right) s_{f}-S\left(\gamma_{0}+\boldsymbol{\theta}_{i}+\boldsymbol{\psi}_{i}\right) z_{f} \\
-S\left(\boldsymbol{\varphi}_{i}+\boldsymbol{\theta}_{i}+\gamma_{0}+\boldsymbol{\psi}_{i}\right) a_{f}+C\left(\gamma_{0}\right) \boldsymbol{x}_{i}=0 \quad(a) \\
z_{0}-z_{P_{i}}-S\left(\gamma_{0}\right) l_{f}-C\left(\gamma_{0}+\boldsymbol{\psi}_{i}\right) s_{f}-C\left(\gamma_{0}+\boldsymbol{\theta}_{i}+\boldsymbol{\psi}_{i}\right) z_{f} \\
-C\left(\boldsymbol{\varphi}_{i}+\boldsymbol{\theta}_{i}+\gamma_{0}+\boldsymbol{\psi}_{i}\right) a_{f}-S\left(\gamma_{0}\right) \boldsymbol{x}_{i}=0 \quad(b) \\
\gamma_{0}+\boldsymbol{\theta}_{i}+\boldsymbol{\psi}_{i}+\boldsymbol{\varphi}_{i}-\gamma_{i}=0 \quad(c)
\end{gathered}
$$

For the hind limbs (i=3...4) :

$$
\begin{aligned}
& x_{0}-\boldsymbol{x}_{P_{i}}-C\left(\gamma_{0}\right) l_{r}-S\left(\gamma_{0}+\boldsymbol{\psi}_{i}\right) s_{r}-S\left(\gamma_{0}+\boldsymbol{\theta}_{i}+\boldsymbol{\psi}_{i}\right) z_{r} \\
& -S\left(\boldsymbol{\varphi}_{i}+\boldsymbol{\theta}_{i}+\gamma_{0}+\boldsymbol{\psi}_{i}\right) a_{r}=0 \quad(d) \\
& z_{0}-z_{P_{i}}+S\left(\gamma_{0}\right) l_{r}-C\left(\gamma_{0}+\boldsymbol{\psi}_{i}\right) s_{r}-C\left(\gamma_{0}+\boldsymbol{\theta}_{i}+\boldsymbol{\psi}_{i}\right) z_{r} \\
& -C\left(\boldsymbol{\varphi}_{i}+\boldsymbol{\theta}_{i}+\gamma_{0}+\boldsymbol{\psi}_{i}\right) a_{r}=0 \quad(e) \\
& \gamma_{0}+\boldsymbol{\theta}_{i}+\boldsymbol{\psi}_{i}+\boldsymbol{\varphi}_{i}-\gamma_{i}=0 \quad(f)
\end{aligned}
$$

\subsection{Determination of Closed-form solutions}

The 12 constraint equations previously presented are non-linear equations. The first step in solving a kinematic problem is to choose enough known variables. This leads to a partitioning of the positional variables between the independent positional variables (inputs) and the dependent positional variables (outputs or the unknowns in other words). The inputs gathered in the vector $\mathrm{p}_{\mathrm{i}}$ chosen were those which are located at the bottom of the appendages because they are the easiest to measure. This input vector $\mathbf{p}_{\mathbf{i}}$ with 13 components is the following for the 4 legs $(i=1 \ldots 4)$ :

$$
\mathbf{p}_{\mathbf{i}}=\left\{x_{P i}, z_{P i}, \gamma_{i}, \varphi_{i}, \theta_{2}\right\}
$$

From ${ }^{[21,23,34]}$, it can be noticed that this choice of inputs is not what is traditionally used in bio-robotics. Indeed, in robotics, the designer usually defines the trajectory of the main body (in the task space) and controls the joint parameters (in the joints or configuration space) located in the legs by using an inverse kinematic model ${ }^{[35]}$. In this paper, the aim of this model is to build a predictive model of the locomotion of an unguligrade quadruped which will permit us to reproduce the dynamics of a quadruped. Therefore, the joint parameters are driven by the movement which have been acquired from the video analysis conducted with the BITS method. The outputs are the remaining parameters and gathered in the output vector $\mathbf{p}_{\mathbf{o}}$ with 12 components and $\mathrm{i}=1 \ldots 4$ :

$$
\mathbf{p}_{\mathbf{o}}=\left\{\theta_{1}, \theta_{3}, \theta_{4}, \psi_{i}, x_{1}, x_{2}, x_{0}, z_{0}, \gamma_{0}\right\}
$$

The inputs are colored in green and the outputs are colored in red. They have been solved by symbolic manipulation in Maple software based on the recognition of the canonical form $A \mathrm{C}($ angle $)+B \mathrm{~S}($ angle $)+C=0$. Several solutions exist but only one is a biologically consistent solution. This solution is defined with the kinematic joints such as a knee joint which has to be well oriented and kept in a restricted range of values. By combining the equations (d.3), (d.4), (e.3), (e.4), equations with the following form are obtained:

$$
A_{1} \mathrm{C}\left(\Theta_{4}\right)+B_{1} \mathrm{~S}\left(\Theta_{4}\right)+C_{1}=0
$$

Where the following variables change has been applied for I:

$$
\Theta_{i}=\left(-\theta_{i}+\gamma_{i}-\varphi_{i}\right) \text { with } i=1 . .4
$$

The close loop between the hind limbs is used to calculate explicitly the angles. By combining (a.2), (b.2), (d.4) and (e.4) equations from the close loops associated with appendages 2 and 4, the positional parameters $\gamma_{0}$ and $x_{2}$ are determined. With (d.3), the parameter $x_{1}$ can be deduced. By combining (c.1) and (c.3) equations from the kinematic loops associated with appendages 1 and 3, the angle $\Theta_{1}$ is determined. The remaining angles' outputs can be then determined. Once all the angles' outputs are determined, the position of central point G1 can be calculated from the two equations among (d.1), (d.2). All the outputs kinematic parameters have been symbolically solved but not detailed 
in the paper for the sake of concision. A model to model check has been conducted. All the calculated outputs determined with Maple software gave the same results than those obtained with the CAD model.

\section{Results}

\subsection{Determination of the positional inputs}

The positional inputs (translational and angular) have been determined with the BITS (implemented through a CAD sketch) and imported images. These definitions of the input data have been extended periodically and they are given with two periods in Fig 11 for the leg 2. The swing and stance phases are identified easily on the positional curves. When $X_{\mathrm{pi}}$ and $\mathrm{X}_{\mathrm{pi}}$ are constant, the foot considered is fixed to the ground and is in a stance phase. When $\mathrm{X}_{\mathrm{pi}}$ grows linearly in function of time and $\mathrm{Z}_{\mathrm{pi}}$ presents a pic, the foot is a swing phase.
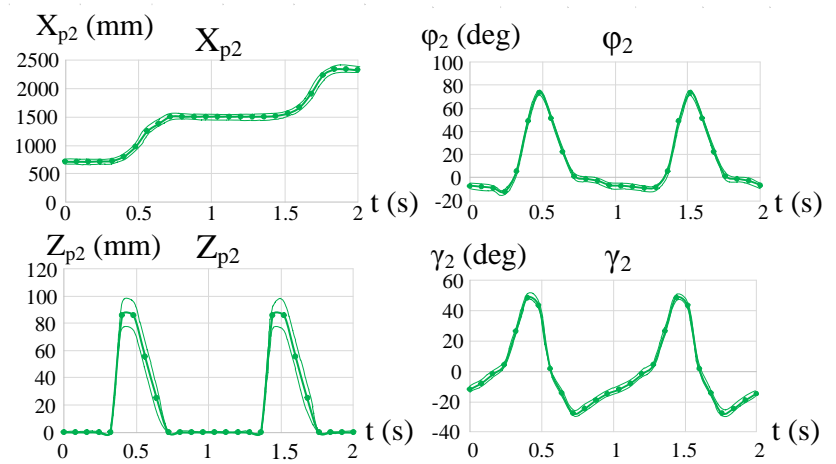

Fig 11 Positional inputs and angular inputs

The periodic signals of the angular inputs have been built by using the cycle period (the sum of a stance phase duration and a swing phase duration) which has been determined on the positional curves. It is notable that the order of magnitude of the amplitude of the wrist $\left(\phi_{i}(t)\right.$ with $\left.\mathrm{i}=1 . .2\right)$ and ankle $\left(\phi_{i}(t)\right.$ with $\left.\mathrm{i}=3 . .4\right)$ angles in Fig 11 is close to the values given in ${ }^{[30,36]}$.

\subsection{Comparison between measured and calculated outputs}

Some outputs, which are possible to determine thanks to the kinematic model, are compared with measurements from pictures. The knee angle $\theta_{4}$ and the position of a central point $\mathrm{G}_{1}$ are given respectively in Fig 12 and Fig 13 . The maximum deviation between the measure and the model for the knee angle is less than $5^{\circ}$. This amplitude and the form of the curve are consistent with the experimental results presented in ${ }^{[30,36]}$.

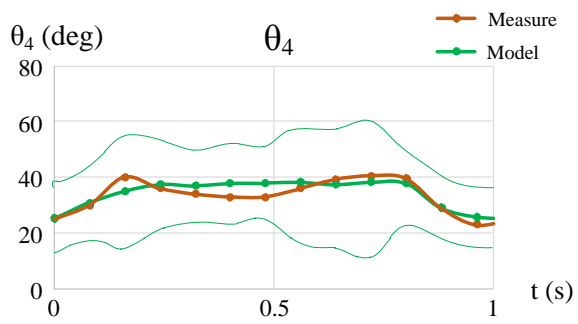

Fig 12 Rear knee output

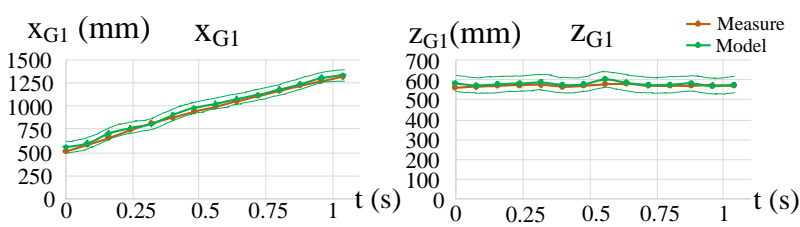

Fig 13 Central point of the main body 
The displacement in the direction of the $\mathrm{x}$ axis is a linear function of time which is explained by the constant speed of the sheep during the experimentation. The maximum deviation for this $x$ component is $67 \mathrm{~mm}$. It is also interesting to note that the vertical displacement along the $\mathbf{z}$ axis stays almost constant. The maximum deviation for this $x$ component is $25.3 \mathrm{~mm}$. The positions for the central point $\mathrm{G}_{1}$ are consistent. These results show a good correlation between the kinematic model and the measurements which have been taken from still-pictures. Some positional parameters for the angle $\theta_{1}$ of the right elbow joint or the displacement $\mathrm{x}_{2}$ between the scapula of the leg 2 and the trunk can be difficult to measure because the vertex of the bones' mechanical axes are hidden behind a thick muscular layer. However, the kinematic model can enable us to give a first estimate of these parameters (Fig 14 and Fig 15). The travel of this angle $\theta_{l}$ is about $60^{\circ}$ which is consistent with the results given in ${ }^{[30,36]}$. The travel of the displacement inferior to 85 $\mathrm{mm}$ also seems physically acceptable. To the author's knowledge, the scapula movement has never been estimated before through a kinematic model ${ }^{[21-23]}$. Further experimentations should be conducted to determine this result and namely the assumption which has been made concerning the sliding direction in the prismatic joint.

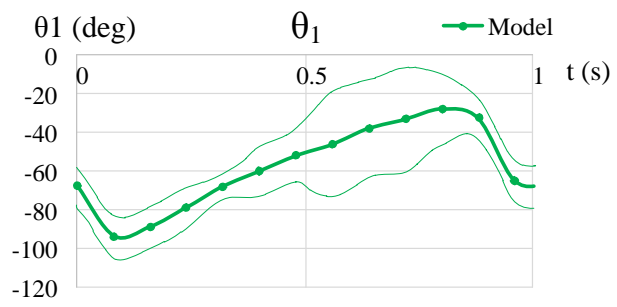

Fig 14 Calculated angle of the left elbow joint

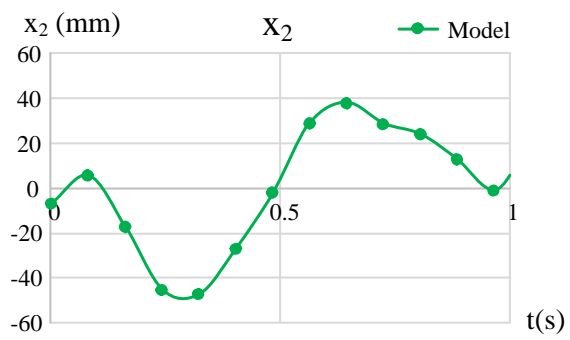

Fig 15 Calculated displacement in the prismatic joint between the scapula of the leg 2 and the trunk

\section{Conclusion}

Within the context of bio-inspiration mechanisms from living beings, low-cost and easy to use processes can be desirable to measure gaits namely in the preliminary design phase. In this sense, a method, to exploit the classic video acquisition system, has been suggested. This method, called the bio-inspired topological skeleton (BITS) method, consists of using a topological skeleton as a pattern in a CAD system to measure kinematic data from pictures. The BITS enables the user to take geometric and topologic constraints into account during the process of measurement. The topological skeleton method has allowed us to find better measurements than those of the standard process. Once the inputs are obtained with this method, the resolution of a quadruped kinematics model has been led symbolically. Certain joints parameters (scapulae movements) have been determined from the kinematic model and compared with experimental data to show the benefits of the method. This method can be extended to 3D skeletons where the thirteen elementary geometric constraints are taken into account from $3 \mathrm{D}$ videos.

\section{Acknowledgement}

This research work received support from the Chair "Bio-inspired mechanical Design” Aix-Marseille University - Airbus Helicopters.

\section{References}

[1] Cappozzo A. Gait analysis methodology. Human Movement Science. 1984, 3, 27-50. 
[2] Marey E J. La machine animale, Germer Baillière, Paris, France, 1873.

[3] Marey E J. De la locomotion terrestre chez les bipedes et les quadrupedes, E. Martinet, Paris, France, 1873.

[4] Muybridge E. Animal locomotion, Da Capo Press., New York, USA, 1969.

[5] Sutherland D H, Hagy J L. Measurement of gait movements from motion picture film. The Journal of bone and joint surgery American volume. $1972, \mathbf{5 4}, 787-797$.

[6] Schmid T, Weishaupt M A, Meyer S W, Waldern N, Peinen K v, Nuss K. High-speed cinematographic evaluation of claw-ground contact pattern of lactating cows. The Veterinary Journal. 2009, 181, 151-157.

[7] Kadaba M, Wootten M, Ramakrishnan H, Hurwitz D, Cochran G. Assessment of human motion with vicon. Proceedings of the Biomechanics Symposium ASME, 1987, 335-338.

[8] Monaghan K, Delahunt E, Caulfield B. Increasing the number of gait trial recordings maximises intra-rater reliability of the CODA motion analysis system. Gait \& Posture. 2007, 25, 303-315.

[9] Jouffroy F, Gasc J. A cineradiographical analysis of leaping in an African prosimian (Galago alleni). In: Jenkins F A, editor. Primate locomotion, Acadamic Press, New York, USA, 1974, 117-142.

[10] Fischer M S, Schilling N, Schmidt M, Haarhaus D, Witte H. Basic limb kinematics of small therian mammals. Journal of Experimental Biology. 2002, 205, 1315-1338.

[11] Fischer M S, Blickhan R. The tri segmented limbs of therian mammals: kinematics, dynamics, and self stabilization-a review. Journal of Experimental Zoology, Part A: Comparative Experimental Biology. 2006, 305, 935-952.

[12] Wehner T, Wolfram U, Henzler T, Niemeyer F, Claes L, Simon U. Internal forces and moments in the femur of the rat during gait. Journal of Biomechanics. 2010, 43, 2473-2479.

[13] Hugel V, Hackert R, Abourachid A. Kinematic modeling of bird locomotion from experimental data. IEEE Transactions on Robotics. 2011, 27, 185-200.

[14] Morris J R W. Accelerometry-A technique for the measurement of human body movements. Journal of Biomechanics. 1973, 6, 729-736.

[15] Muro-de-la-Herran A, Garcia-Zapirain B, Mendez-Zorrilla A. Gait analysis methods: an overview of wearable and non-wearable systems, highlighting clinical applications. Sensors. 2014, 14, 3362-3394.

[16] Govoni V, Rahal S C, Agostinho F, Conceição R, Tsunemi M, El-Warrak A. Goniometric measurements of the forelimb and hindlimb joints in sheep. Veterinary and Comparative Orthopaedics and Traumatology. 2012, 25, 297-300.

[17] Richards J G. The measurement of human motion: A comparison of commercially available systems. Human Movement Science. 1999, 18, 589-602.

[18] Bar-Cohen Y. Biomimetics--using nature to inspire human innovation. Bioinspiration \& biomimetics. $2006,1, \mathrm{P} 1$.

[19] Hedrick T L. Software techniques for two-and three-dimensional kinematic measurements of biological and biomimetic systems. Bioinspiration \& biomimetics. 2008, 3, 034001.

[20] Clément A, Rivière A, Serré P. Geometry and topology declarative: a new paradigm for CAD-CAM systems. Proceedings of IDMME, 1998, 587-595.

[21] Semini C, Tsagarakis N G, Guglielmino E, Focchi M, Cannella F, Caldwell D G. Design of HyQ-a hydraulically and electrically actuated quadruped robot. Proceedings of the Institution of Mechanical Engineers, Part I: Journal of Systems and Control Engineering. 2011, 225, 831-849.

[22] Spröwitz A, Tuleu A, Vespignani M, Ajallooeian M, Badri E, Ijspeert A J. Towards dynamic trot gait locomotion: Design, control, and experiments with Cheetah-cub, a compliant quadruped robot. The International Journal of Robotics Research. 2013, 32, 932-950.

[23] Hyun D J, Seok S, Lee J, Kim S. High speed trot-running: Implementation of a hierarchical controller using proprioceptive impedance control on the MIT Cheetah. The International Journal of Robotics Research. 2014, 33, 1417-1445.

[24] Nam W, Seo T, Kim B, Jeon D, Cho K-J, Kim J. Kinematic analysis and experimental verification on the locomotion of gecko. Journal of Bionic Engineering. 2009, 6, 246-254.

[25] Boyer F, Porez M. Multibody system dynamics for bio-inspired locomotion: from geometric structures to computational aspects. Bioinspiration \& biomimetics. 2015, 10, 025007.

[26] Linzey D. Vertebrate Biology, McGraw-Hill Press, New York, USA, 2001.

[27] Moissenet F, Chèze L, Dumas R. A 3D lower limb musculoskeletal model for simultaneous estimation of musculo-tendon, joint contact, ligament and bone forces during gait. Journal of Biomechanics. 2014, 47, 50-58.

[28] Clément A, Rivière A, Serré P, Valade C. The TTRSs : 13 Constraints for Dimensioning and Tolerancing. In: ElMaraghy H A, editor. Geometric Design Tolerancing: Theories, Standards and Applications, Springer US, Boston, MA, 1998, 122-131.

[29] Mermoz E, Linares J-M, Bernard A. Benefits and limitations of parametric design implementation in helicopter gearbox design phase. CIRP Annals-Manufacturing Technology. 2011, 60, 199-202.

[30] Abourachid A, Laville E. Kinematic study of the locomotion of two crossbreds of lambs. Annales de Zootechnie. 1997, 46, $219-230$. [31] Lartigue C, Bourdet P, Mathieu L, Mehdi-Souzani C. Algorithms for the calibration of laser-plane sensors on CMMs. Advanced Mathematical And Computational Tools In Metrology VI, World Scientific, Torino, Italy, 2004, 82-97.

[32] Ricolfe-Viala C, Sánchez-Salmerón A-J. Robust metric calibration of non-linear camera lens distortion. Pattern Recognition. 2010, 43, 1688-1699.

[33] BIPM, IEC, IFCC, ISO, IUPAC, IUPAP, OIML. Guide to the expression of the uncertainty in measurement. norme XP X 07-020; 1996.

[34] Hugel V, Blazevic P. Towards efficient implementation of quadruped gaits with duty factor of 0.75. Proceeding of the International IEEE Conference on Robotics and Automation 1999, 2360-2365.

[35] Son D, Jeon D, Nam W C, Chang D, Seo T, Kim J. Gait planning based on kinematics for a quadruped gecko model with redundancy. Robotics and Autonomous Systems. 2010, 58, 648-656.

[36] Faria L G, Rahal S C, Agostinho F S, Minto B W, Matsubara L M, Kano W T, Castilho M S, Mesquita L R. Kinematic analysis of forelimb and hind limb joints in clinically healthy sheep. BMC Veterinary Research. 2014, 10, 1. 